\title{
L4 PIEDRA NOIRE AU TONKIN ET EN ANNAM
}

\section{Par DANG-VAN-NGU}

Alors que les piedras blanches ont été observées aussi bien en Europe et en Asie que dans le Nouveau Monde, on a cru, pendant longtemps, que la piedra noire était une affection spéciale à l'Amérique du Sud.

Bien que le diagnostic des piedras soit extrêmement facile, car les nodules sont bien visibles et sensibles à la palpation, il semble que les piedras noires n'aient été rencontrées pour la première fois dans l'Ancien Monde qu'en 1936. A cette date, Kuypers signale, en effet, dans l'ouest de Bornéo, vingt cas de piedra, dont dix-huit piedras blanches, dues au Trichosporum giganteum, et deux piedras noires, paraissant identiques aux nodules brésiliens à Piedraia hortai.

Un an plus tard, en 1937, Souchard et Nguyen-Van-Huong font connaitre l'existence, en Cochinchine, de nombreux porteurs de nodosités piedriques noires, en tout semblables, macroscopiquement et microscopiquement, à celles de la piedra noire brésilienne.

En 1938, Boedyn et Verbunt ont étudié deux cas de pietra noire provenant du centre de Java et ont cru devoir décrire une espèce nouvelle sous le nom de Piedraia javanica Boedyn et Verbunt 1938. Ces auteurs pensent que la piedra noire doit être fréquente à Batavia, mais qu'elle est méconnue à cause du peu de visibilité des nodosités sur les cheveux très pigmentés.

Nous-même avons retrouvé cette affection en Annam et au Tonkin.

En mars 1939, un élève d'une école privée de Hué nous est envoyé pour examen ses propres cheveux, récemment atteints d'une affection nodulaire. A cette époque, environ $25 \%$ des élèves de l'école étaient atteints de la même affection. La source de contagion était probablement un élève cochinchinois venant de quitter sa province.

Rapidement la maladie prit une grande extension et bientôt l'affection a pu être décelée dans toutes les écoles de garçons de Hué: Il est digne de remarque qu'aucun cas n'a été signalé jusqu'ici dans l'école des jeunes filles.

En avril 1939, nous avons de nouveau rencontré cette affection,

Annales de Parasitologie, T. XVII, No $4 .-1^{\text {er }}$ juillet 1939, p. 359-363. 
cette fois à Hanoï. Les cheveux provenaient d'un étudiant en droit n'ayant jamais quitté Hanoï, mais cohabitant avec d'autres camarades cochinchinois porteurs de nodosités piedriques. Une enquête faite parmi les étudiants en médecine nous a vite permis de réunir ane dizaine de cas semblables. Les cas les plus anciens datent de deux ans et les porteurs en sont toujours des étudiants cochinchinois du P.C.B. ou de $1^{\text {re }}$ année, ayant donc contracté l'affection dans un lycée de Saïgon. Les cas récents trouvent toujours ces derniers comme source de contagion.

L'aspect de tous les cheveux malades était caractéristique de la piedra noire : les nodules étaient très durs et très adhérents au cheveu. Ils étaient tellement opaques que souvent la potasse seule n'arrivait pas à les éclaircir suffisamment, l'action combinée de la potasse et du chloralactophénol étant alors nécessaire.

La plupart de ces nodules ont la forme tronconique, mais beaucoup sont irrégulièrement cylindriques ou fusiformes (pl. XXVI, fig. 1). Leur longueur ne dépasse pas $1 \mathrm{~mm} .5$ et leur largeur $0 \mathrm{~mm} .5$. Les nodules complètement développés sont souvent aplatis transversalement, de sorte que leur section perpendiculaire à l'axe du cheveu apparaît elliptique. Ils siègent sur la couche de cheveux la plus superficielle de la région occipitale, cheveux dont le point d'implantation est dans la région du sommet de la tête. Le nombre de nodules est très variable d'un cheveu à l'autre, il peut y en avoir de un à une vingtaine. La plupart du temps, il y en a deux ou trois par cheveu. Quand un cheveu porte un seul nodule, celui-si siège fréquemment à quelques centimètres de l'extrémité libre, très loin du point d'implantation. Les nodules suivants se développent de plus en plus près de la racine du cheveu; l'infection semble donc débuter par la périphérie et gagner la base du cheveu de proche en proche, à la faveur peut-être des stolons décrits par Brumpt et Langeron (1934), quoique nous n'ayons pas pu nous-mêmes observer ces formations.

Après l'action de la potasse ou du chloralactophénol, le nodule apparaît constitué par des filaments à directions radiaires, formés d'éléments polyédriques réunis par un ciment de couleur brunjaunâtre. Les asques sont toujours disposés perpendiculairement à l'axe du cheveu. Leur plus grand diamètre varie de 30 à $50 \mu$ (en moyenne $44 \mu 8$ ) et leur petit diamètre de 25 à $30 \mu$ (moyenne $31 \mu 6$ ). Ils renferment tous 8 ascopores légèrement incurvées en $S$, de $38 \mu$ de long sur $6 \mu$ de large, portant des filaments polaires de 8 à $10 \mu$ de long. Ces aspects correspondent aux descriptions de Piedra hortai (1).

(1) La grande dimension des asques et la brièveté relative des filaments polaires tendent en particulier à les rapprocher de l'aspect décrit par Brumpt et Langeron sur le matériel communiqué par le docteur Alonso Restrepo, de Medellin. 
Les cultures faites avec ce matériel nous ont donné d'ailleurs des cultures noires caractéristiques au bout de cinq jours.

Sur les coupes transversales de cheveux piédriques, nous avons pu retrouver tous les aspects décrits par Brumpt et Langeron à propos du soulèvement de la cuticule épidermique, celle-ci pouvant se recroqueviller sur elle-même ef loger, dans sa concavité, des asques, simulant ainsi de véritables périthèces.

Nous avons cependant constaté souvent que les filaments mycéliens, après avoir soulevé l'épidermicule, peuvent s'insinuer entre les fibriles du cheveu et les décoller en lamelles concentriques à la surface. Parfois, les filaments gagnent directement la moelle du cheveu en suivant une fissure radiaire. Les lésions n'ayant aucune action sur la continuité des fibrilles ne tendent nullement à rendre fragile le cheveu qui, soumis à la traction, ne se casse jamais au niveau du nodule.

Toutes les affections nodulaires des cheveux sont réunies sous le nom de piedra. Langeron distingue deux groupes de piedras bien distincts au point de vue clinique, épidémiologique et parasitologique : les piedras blanches et les piedras noires.

Les piedras blanches forment des nodules plus ou moins colorés, mais jamais noirs et opaques, ni très durs. Elles sont dues à diverses espèces de champignons hyphomycètes du genre Trichosporum, caractérisé par l'aspect plissé et membraneux des colonies, par la ramification caractéristique du mycélium donnant toujours des rameaux immédiatement au-dessus d'une cloison et enfin par la présence, à la surface des supports compacts, d'organes spéciaux de fixation en forme de chou-fleur (appressorium). L'aire de dispersion des piedras blanches est très étendue : on les rencontre en Europe, aux Indes, au Japon, en Amérique du Sud.

Les piedras noires sont caractérisées par des nodules toujours de couleur très foncée, opaques et très durs. Elles sont dues à des champignons du genre Piedraia Da Fonseca et Leão 1928. La structure des nodules a été bien des fois décrite depuis le mémoire fondamental d'Horta (1911) : ils sont formés d'un pseudo-parenchyme ou ascostroma, constitué par des files d'éléments polyédriques à disposition radiaire et renfermant des cavités, en forme de conceptacles, contenant des spores allongées, non cloisonnées, terminées aux deux extrémités par un filament. Le nombre de ces ascospores peut être de 4 ou de 8 , suivant l'espèce. Langeron a démontré, en 1929, que les Piedraia sont des ascomycètes pyrénomycètes appartenant au groupe biologique des astérinées.

Au Brésil, où elle a été si bien étudiée par Horta en 1911, on rencontre la piedra noire tout le long du littoral atlantique, depuis 
Bahia, au Nord, jusqu’à Rio Grande do Sul, au Sud ; à Cuyaba et à Bello-Horizonte.

Elle a été signalée ensuite au Paraguay, à Asuncion, par Migone et Cavazzoni, puis par Delamarre et Gatti ; en Uruguay, par Barboza à Minas de Corrales, tout à fait à la frontière du Brésil ; en Argentine, à Buenos-Aires, par C. Orol Arias, Parodi et Orol Arias et par F. Niño ; en Guyane Hollandaise, à Paramaribo, où Ch.-G. Aars a étudié 60 cas; en Guyane britannique, le cas décrit par J.-H. Macleod en 1912 semble devoir être rangé aussi dans la piedra noire ; en Colombie, la première observation paraît, d'après Langeron (1936), être celle de Desenne (1878). Récemment, des cas ont été observés en 1933 par Peña Chavarria en des points très variés ; Brumpt et Langeron (1934) ont décrit un cas à Medellin d'après le matériel envoyé par Alonso Restrepo.

Tous ces cas, rencontrés et décrits jusqu'en 1934 en Amérique du Sud, sont causés par Piedraia hortai et possèdent des asques à huit ascospores, munies de filaments polaires plus ou moins allongés. Les légères différences constatées par divers auteurs dans la morphologie des ascostromas et des cultures n'ont pas paru suffisantes à Brumpt et Langeron (1934) pour maintenir les autres noms spécifiques qui avaient été proposés. Par contre, ces auteurs, ayant étudié un matériel remis par le $\mathrm{D}^{r}$ Machado, de Caracas (Venezuela), ont décrit une nouvelle espèce qu'ils ont nommée Piedraia venezuelensis. Outre l'aspect des nodules, qui sont fusiformes au lieu d'être tronconiques comme ceux de Piedraia hortai, $P$. venezuelensis est caractérisé surtout par des asques ne renfermant que quatre ascospores. De plus, ces ascospores sont trapues, à paroi épaisse et présentent, à la place des filaments polaires, deux pointes épaisses et courtes.

Les observations de Souchard et Nguyen-Van-Huong et les nôtres, établissant l'existence de cette affection dans différentes villes d'Indochine (Saïgon, Hué, Hanoï), montrent qu'elle peut s'étendre à d'autres régions encore. Elles viennent confirmer l'opinion de Langeron (1929 et 1936) concernant la stricte affinité des piedras noires pour. les régions très humides (climat astérinéen d'Arnaud). L'Indochine française est, en effet, une presqu'île saturée d'humidité. Cette humidité est fournie par la mer qui la baigne sur toute sa longueur; elle est entretenue par les immenses forêts de la chaîne annamitique et périodiquement augmentée par la mousson du Sud-Est. Notons qu'à Hué, en particulier, il tombe plus de deux mètres de pluie par an, aussi l'affection, sporadique à Hanoï et à Saïgon, y a-t-elle pris, en profitant de la coïncidence avec la saison des pluies, une allure épidémique. 
ANNALES DE PARASITOLOGIE

T. XVII, No 4, 1er Juillet 1939
Planche XXVI

(Mémoire DANG-VAN-NGC)
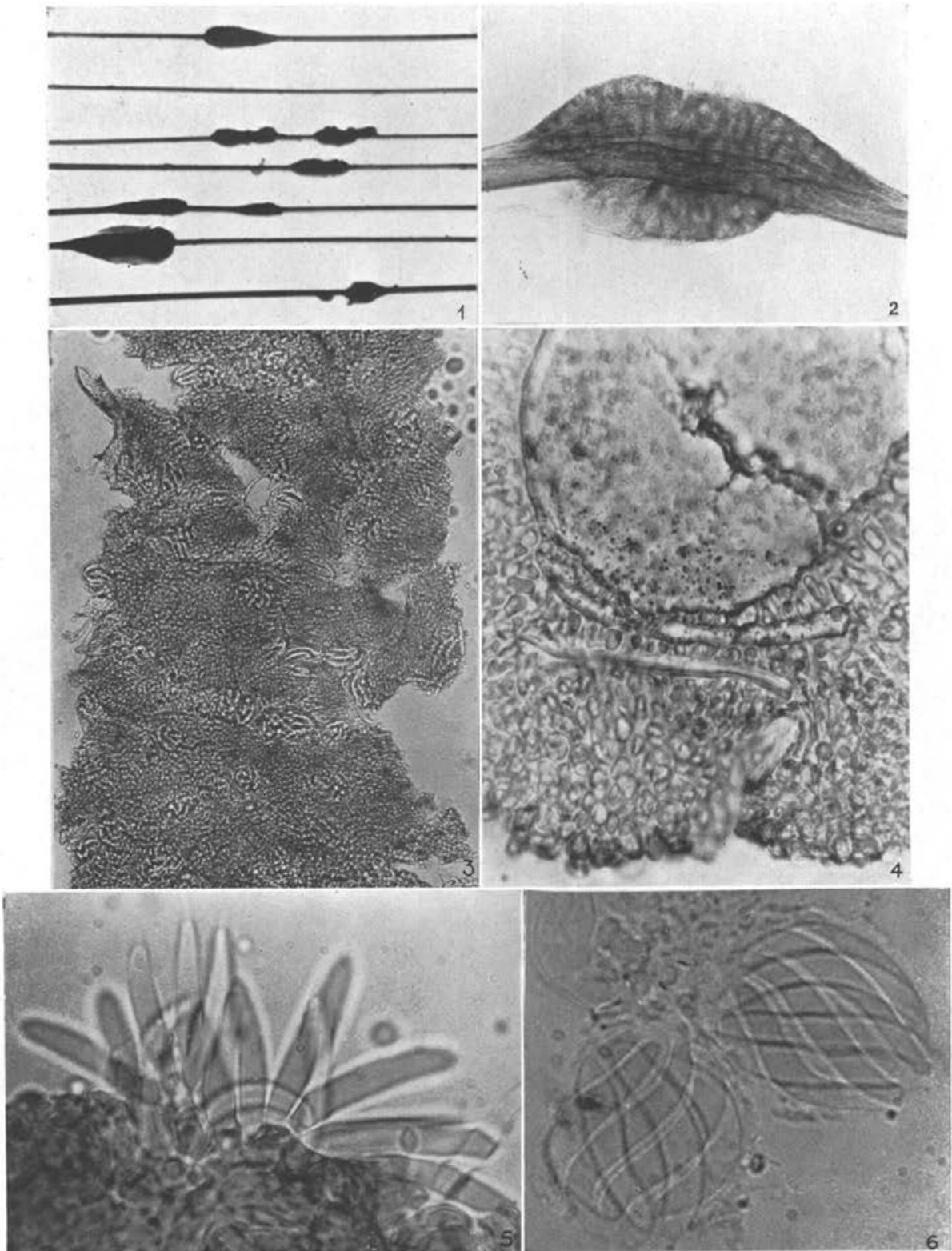

Masson et Cie, Editeurs 



\section{RÉSUMÉ}

De nombreux cas de piedra noire ont été rencontrés à Hué et à Hanoï, et cette affection existe à Saïgon depuis au moins deux ans. Le climat de l'Indochine est très favorable à l'évolution de ces champignons parasites.

L'aspect des nodules et la culture permettent de les identifier à Piedraia hortai. Sur les coupes, nous avons constaté que le mycélium, après avoir décollé l'épidermicule, s'insinue souvent entre les fibrilles du cheveu, soit parallèlement, soit perpendiculairement à sa surface.

\section{Bibliographie}

Boedyn (K. B.) et Verbunt (J. A.). - Annotations about dermatomycoses in Batavia. Mycopathologia, I, 1938, p. 196-198, pl. XXIX-XXXI.

Brumpt (E.) et Langeron (M.). - Considérations sur la piedra de l'Amérique du Sud à l'occasion d'un cas provenant du Venezuela. Description d'une espèce nouvelle : Piedraia venezuelensis n. sp. Ann. de Paras., XII, 1934, p. $134-161$.

Langenon (M.). - Les astérinées parasites de l'homme. La piedra. Ann. de Paras., VII, 1929, p. 309-324.

Piedra. Nouvelle Pratique dermatologique, II, 1936, p. 377-408.

KuYpers (C. A.). - Piedra in West Borneo. Geneesk. Tijdschr. Nederl. Indië, LXXVI, 1936, p. 2344-2356.

Souchard et Nguyen-Van-Huong. - La piedra noire de Cochinchine. Ann. de Paras., XV, 1937, p. 539-543.

\section{Laboratoire de Parasitologie de l'Ecole de Médecine d'Hanö̈}

(Directeur : Prof. H. Galliard).

\section{EXPLICATION DE LA PLANCHE XXVI}

Fig. 1. - Divers aspects des nodules piédriques. $\times 10$.

FIG. 2. - Nodule avec nombreux asques apparaissant sous forme de taches claires sur le fond sombre de l'ascostroma. $\times 42$.

Fig. 3. - Ascostroma dissocié dans la potasse : asques et ascospores très visibles. $\times 290$.

FIG. 4. - Coupe transversale d'un cheveu piédrique. Décollement de l'épidermicule. Envahissement de la zone sous-cuticulaire ; fissuration radiaire du cheveu par envahissement du mycélium. $\times 740$.

Fig. 5. - Eclatement des asques à la périphérie d'un nodule. $\times 740$.

FIG. 6. - Divers apects d'asques. $\times 740$. 